\title{
Diferentes tipos de incidencia de los procesos cognitivos de revisión sobre la coherencia de textos narrativos: un estudio con niños de 10 años"
}

\section{Different types of incidence that revision cognitive processes have on narrative texts coherence: a research with ten-year-old children}

Recibido: junio 10 de 2010 | Revisado: enero 19 de 2011 | Aceptado: marzo 16 de 2011

\author{
DiANA De CASTRO** \\ MirAlBA CORREA ${ }^{* * * *}$ \\ Universidad del Valle, Cali, Colombia
}

SICI: 1697-9267(201206)11:2<441:DTIPCR>2.0.TX;2-J

Para citar este artículo. De Castro, D. \& Correa, M. (2012). Diferentes tipos de incidencia de los procesos cognitivos de revisión sobre la coherencia de textos narrativos: un estudio con niños de 10 años. Universitas Psychologica, 11(2), 441-454.

Artículo derivado de la tesis de Maestría en Psicología titulada "Los procesos cognitivos de revisión en el establecimiento de la coherencia en narraciones escritas por niños de 10 años" de la Universidad del Valle. Se reconocen y agradecen los valiosos aportes como asesor estadístico a Yilton Riascos, candidato a doctor (Universidad del Valle), y la guía y contribuciones en el análisis semiótico de Nicole Everaert- Desmedt PhD (Universidad Saint-Louis, Bruselas).

** Instituto de Psicología. Ciudad Universitaria Meléndez. Edificio 385, Piso 4. E-mails: dipadeda@yahoo.comódiana.decastro@correounivalle. edu.co. ResearcherID: De Castro, D. F-3575-2012.

**** Instituto de Psicología. Ciudad Universitaria Meléndez. Edificio 385, Piso 4. E-mail: miralbacorrea@yahoo.com. ResearcherID: Correa, M. F-4024-2012.

\section{RESUMEN}

Esta investigación se enmarca en la Psicología del Desarrollo Cognitivo, su propósito es identificar los procesos de revisión en las reescrituras elaboradas por niños de 10 años y su incidencia en el establecimiento de la coherencia de textos narrativos. El análisis cualitativo y estadístico de tres producciones escritas y dos conversatorios de 30 niños de 10 años, permite identificar tanto los diferentes tipos de incidencia que tiene la revisión sobre la coherencia, como las trayectorias que siguen los niños para lograr transformaciones a nivel estructural y discursivo. El análisis de los cambios que los niños realizan en sus producciones escritas, permite conocer las transformaciones en la coherencia y da cuenta de avances, retrocesos, oscilaciones y permanencia en los desempeños. Finalmente, se identifican funcionamientos mentales decisivos en el establecimiento de la coherencia y dimensiona la importancia de propuestas educativas que focalicen en la revisión.

Palabras clave autores

Coherencia, escritura, procesos cognitivos de revisión, textos narrativos.

Palabras claves descriptores

Cognición, niños, lenguaje escrito.

\section{A B S T R A C T}

The following research stands within the framework of cognitive development psychology. Its purpose is to identify how the revision process works in the re-writings done by ten-year-old children, and the incidence this has on establishing coherence in narrative texts. The qualitative and statistical analysis of three written productions and two conversations held with 30 ten-year-old children enables to identify the different types of incidence that revision has over coherence, as well as the paths followed by children to attain transformations at both structural and discursive levels. Analysis of children's changes in their written productions allows noticing transformations in coherence and it also helps identify the advances, retreats, oscillations, and permanence in the performances. Finally, mental functionings decisive for coherence are identified; the article ends up pointing out the importance of having educational proposals that focus on revision.

Key words authors

Coherence, writing, revision cognitive processes, narrative texts.

Key words plus

Writing process, children, composition. 
En las últimas décadas la Psicología muestra un interés particular en estudiar los funcionamientos mentales involucrados en la producción textual. Es en esta perspectiva que Bereiter y Scardamalia (1987, 1992) proponen la psicología de la composición escrita, que estudia los procesos cognitivos puestos en marcha al elaborar un texto, identificando entre ellos la revisión, entendida como la más importante y constitutiva de la naturaleza de la escritura (Bereiter \& Scardamalia, 1987, 1992; Camps, 1992; Cassany, 1996; Allal \& Chanquoy, 2004). Camps (1992) afirma que la revisión es el proceso más importante dentro de la redacción, al considerarla propia de la construcción textual. Bereiter y Scardamalia (1987) enfatizan que la revisión se constituye en un proceso característico de la composición escrita, que la distingue de la conversación. En la escritura es posible volver sobre los contenidos y modos discursivos tal como han sido propuestos, lo que facilita su análisis y la objetivación de las decisiones tomadas por el autor, mientras en la conversación se trata de responder más a las señales, los gestos e intervenciones del interlocutor (Blanche-Benveniste, 1998; Olson, 1998; Ong, 1999).

Los procesos de revisión son inherentes a la composición escrita y no solo se ponen en marcha como respuesta a errores, disonancias y disarmonías. También implican los funcionamientos que los niños realizan al reconsiderar su propio texto y hacer los cambios que estiman necesarios cuando reelaboran sus propias narraciones. Si bien se conoce que la revisión puede operar sobre los planes y sobre el escrito cuando se está elaborando, en la presente investigación se estudian los procesos de revisión después de una primera escritura -revisión diferida-. Ligadas al interés en los procesos de revisión, surgen las preguntas sobre cómo se establece y transforma la coherencia de los textos narrativos escritos por los niños, constituyéndose estas en las dos temáticas que se articulan.

La decisión de trabajar con textos narrativos se deriva de que son conocidos por los niños a más temprana edad y de forma más natural. Ellos reconocen la estructura de las narraciones, proponen por sí mismos un tópico para su historia, unos perso- najes y eventos que se desarrollan (Bigas et al., 1994; Orozco \& Correa, 2001; Orozco, Correa, Conde \& Hurtado, 2002; Teberosky, 1989). Las narraciones se despliegan sobre las vicisitudes humanas, por lo que los niños cuentan con el conocimiento sobre el mundo que les permite crear narraciones propias (Bruner, 1988). Por consiguiente, se espera que dispongan de una mayor flexibilidad en su posibilidad de construir historias y posiblemente de transformarlas durante la revisión, a diferencia de lo que sucedería con otros tipos de textos, como el expositivo.

Los textos narrativos escritos por los niños revelan cómo han organizado sus conocimientos sobre las narraciones constituidas por ellos. De allí, el interés por estudiar la coherencia, entendida como la forma cómo los eventos o las diferentes partes de la historia están interrelacionados, dándole una organización y una unidad a lo que se cuenta (Shapiro \& Hudson, 1991, 1997 citados por Cain, 2003; Martínez, 1994). De este modo, la coherencia implica la estructura global de los eventos en la narración y el conjunto de relaciones que se establecen cuando el narrador lleva a cabo el relato. Así, la coherencia responde a un funcionamiento mental del sujeto, que exige el establecimiento de un tópico, el reconocimiento de un objeto de valor sobre el que se articulan los eventos narrados y una estructura que permita ligar los sucesos, al igual que una congruencia de los comportamientos, estados mentales, estados anímicos y características psicológicas de los personajes.

La coherencia y los procesos de revisión han sido abordados de forma separada (Bereiter \& Scardamalia 1987, 1992; Cain, 2003; Calsamiglia \& Tusón, 1999; Camps, 1992; Cassany, 1997; De Vega \& Cuetos, 1999; Graham, Harris \& Mason, 2005; Hickmann \& Schneider, 2000; Levy, 2003; McCutchen, 1995; Miras, 2000); al estudiarse de manera articulada, permiten un mayor avance en la comprensión de los funcionamientos mentales de los niños. Desde esas consideraciones, se plantea la pregunta de investigación: ¿De qué manera inciden los procesos de revisión en la transformación de la coherencia en narraciones escritas por niños de 10 años? En este sentido, los resultados del estudio 
buscan identificar los procesos de revisión en las reescrituras propuestas por el niño y su incidencia en el establecimiento de la coherencia de textos narrativos escritos.

La investigación propuesta se sitúa en una perspectiva narrativa en la que se privilegian los aspectos semánticos ligados con la coherencia textual. Las propuestas de trabajo y el análisis se hacen sobre textos completos y no sobre fragmentos de texto. Esta postura se contrapone a los estudios desde el procesamiento de la información, en los que el análisis se hace sobre fragmentos de textos o tareas no contextualizadas (Bortolussi \& Dixon, 2003; Bourdin \& Fayol, 2002; Flower \& Hayes, 1996; Severinson Eklundh \& Kollberg, 1996).

La investigación se inscribe en una perspectiva de la cognición en la que se indaga sobre la forma como los niños reelaboran sus textos en periodos cortos de tiempo, lo que permite estudiar sus funcionamientos mentales en la escritura y en la reelaboración de sus narraciones. El análisis del funcionamiento del niño estudiándolo por sí mismo es una perspectiva que se empieza a desarrollar. En este estudio, se toma como punto de referencia las producciones del mismo niño, lo que permite seguir el proceso de escritura en su construcción. Con este fin, se realiza un seguimiento de las distintas versiones, de los aspectos que capturan el interés y la atención, así como aquello que los niños como autores consideran adecuado agregar, cambiar $\mathrm{u}$ omitir. El diseño propuesto es uno de los aportes que ofrece el presente trabajo.

\section{Método}

\section{Participantes}

En la investigación participaron 30 niños y niñas de diez años que cursan quinto grado de primaria de una escuela pública de la ciudad de Cali. Los participantes fueron seleccionados a través de muestreo aleatorio. La muestra estuvo conformada por 3 producciones escritas y 2 conversatorios de cada sujeto, lo que permite un seguimiento de la construcción textual. Se escogió la edad de 10 años, porque es garantía de un mayor dominio del código escrito.

\section{Instrumentos y materiales}

Se propone a los niños una tarea de escritura constituida por una imagen con dos enunciados generativos -fuente generativa- (véase Apéndice), herramienta que les posibilita entrar en la ficción y empezar a considerar mundos posibles en los que se desenvuelva su historia (veáse De Castro, 2008 para un análisis comparativo de diferentes tareas de escritura). La imagen y los enunciados generativos son una adaptación de una obra de Van Allsburg (1999).

Las consignas de escritura propuestas a los niños para la composición, explicitan los siguientes aspectos: (a) tendrán lectores reales, dado que muchos niños leerán su cuento; (b) se trata de lectores competentes, es decir, no deben bajar la calidad narrativa en un intento de realizar un texto simple para un lector poco preparado; (c) la consigna los sitúa como escritores competentes, pueden y saben hacer; (d) la escritura tiene un fin: ayudar a aprender a escribir a otros niños y (e) los lectores no conocen la imagen a partir de la que construyen el cuento, solo tienen acceso a la historia a través del escrito.

\section{Procedimiento}

Para la escritura de los textos se trabaja con cada niño de manera individual durante cuatro sesiones en días diferentes. La primera sesión corresponde a la fase de familiarización que contempla una conversación con el niño sobre los cuentos que conoce y la escritura de una producción narrativa realizada desde una fuente análoga a la utilizada para la recolección de datos, que consiste en una imagen con dos enunciados generativos. En la segunda, el niño elabora su narración escrita a partir de la fuente generativa, otra imagen con dos enunciados (Escritura 1).

En la tercera sesión, el niño lee mentalmente la narración que escribió, para que la recuerde y restituya una representación total del texto. Luego, el niño vuelve a leer mentalmente su narración y señala los aspectos que, según su propio criterio, es adecuado cambiar para mejorarla y para que otros niños lectores de su cuento puedan comprenderla 
mejor. En este momento, el investigador acompaña al niño en la mesa, lo que permite asegurarse de su lectura mental, a través de indicios como el movimiento ocular, el seguimiento del texto con su lapicero o con el dedo y la escritura de señas -puntos, guiones, asteriscos, subrayados, etc.- que resaltan los aspectos que el niño desea cambiar en la composición. En la investigación se proporcionan a los participantes lápices de diferentes colores según el momento del procedimiento, para identificar cuándo se producen las señas o cambios sobre el texto.

Posteriormente, el niño lee su texto en voz alta al mismo tiempo que realiza un conversatorio con el investigador sobre los enunciados señalados, las razones para seleccionarlos y los cambios que haría. Finalmente, realiza las transformaciones que considera necesarias en su escrito al reescribir su narración (Reescritura 2). Estos pasos se plantean de la misma forma para la tercera escritura en la cuarta sesión (Reescritura 3). Durante el proceso, los niños tienen a su disposición su escrito anterior; de esta forma se controla los efectos que podría tener la memoria, si los niños tuvieran que recordar sus textos.

Las producciones escritas se recogen, los conversatorios se graban en audio y se transcriben. Para el análisis de esta información, se construye un instrumento de análisis en el que se establecen las categorías y se desglosan en subcategorías e indicadores (De Castro, 2008). Las categorías estudiadas sobre las que se hace el seguimiento durante el proceso de composición textual son: 1) desarrollo de un tópico; 2) estructura del texto; 3) caracterización de los personajes y 4) enlaces de las situaciones a través de la articulación del plano de la acción y de la conciencia que alude al establecimiento de relaciones entre las acciones o situaciones - plano de la acción-, y los pensamientos, sentimientos, deseos, conocimientos, intenciones de los que intervienen en la acción -plano de la conciencia- (Bruner, 1988). Los conversatorios complementan la información que proporcionan los textos escritos, al favorecer la identificación de los criterios que construyen los niños, para establecer los marcos de revisión y las alternativas de corrección consideradas.
De este modo, se inicia con un análisis cualitativo del proceso de composición textual, en el que se realiza un seguimiento desde la primera escritura, el conversatorio, la segunda escritura, el segundo conversatorio hasta la tercera escritura para cada niño de los cambios, reflexiones y comentarios sobre el texto en función de las cuatro categorías señaladas. El punto de referencia en el estudio son las producciones previas y el propio proceso de cada sujeto.

Una vez realizado este análisis particular de cada proceso para cada niño, se realiza un análisis estadístico a través de tablas de clasificación múltiple, para establecer agrupaciones desde las trayectorias en los desempeños de los participantes durante el proceso de escritura, con el fin de identificar tipos de incidencia de la revisión sobre la coherencia. Con el fin de continuar con una clasificación de los desempeños y determinar la similitud entre los conjuntos de variables categóricas de forma que se valide la objetividad de la clasificación, se ejecuta un análisis de correlación canónica no lineal desde el programa SPSS. El análisis de correlación canónica no lineal permite conocer cómo se asocian los sujetos y trabajar con conjuntos de variables cualitativas; en este caso, los desempeños textuales para cada una de las categorías que dan cuenta de la coherencia.

Los resultados de los análisis cualitativos y estadísticos se cotejan, de modo que se enriquece la comprensión de los procesos de composición de cada sujeto y los elementos comunes en la forma de proceder que hacen posible la clasificación y la identificación de distintos tipos de incidencia de la revisión sobre la coherencia de la narración.

\section{Resultados}

El análisis de los datos permite identificar cuatro tipos de incidencia de los procesos de revisión, que concluyen en reescrituras sobre la coherencia en textos narrativos: (a) Incidencia positiva sobre la coherencia: alude a los cambios realizados en la segunda y tercera reescritura derivados de procesos de revisión efectuados por los niños que los llevan a transformar partes de su texto para precisar, aclarar, desarrollar y relacionar aspectos propuestos 
en la historia; (b) Incidencia oscilatoria sobre la coherencia: se refiere a transformaciones que tienen diferentes efectos sobre el texto en una misma producción o al considerar diferentes reescrituras, así se presentan cambios en los que el niño muestra un avance en el establecimiento de la coherencia -precisa, aclara, desarrolla o relaciona aspectos propuestos en la historia- y modificaciones que influyen negativamente en ella -propone otros contenidos que resultan confusos, incongruentes, desarticulados o al margen del tópico- (c) Incidencia local: define los cambios en aspectos particulares que mantienen la organización de la versión previa, así las reescrituras se caracterizan por cambios a nivel del código escrito y (d) Incidencia negativa sobre la coherencia: alude a los procesos de revisión que llevan a realizar cambios para la segunda y tercera producción en los que se suprimen los enlaces del texto, el desarrollo de situaciones que explican un determinado suceso y se incluye información al margen del tópico de la narración.

La Figura 1 presenta la distribución del grupo de acuerdo con el nivel de incidencia. Esta distribución muestra que la mitad de los niños logran realizar cambios, para avanzar en el establecimiento de la coherencia en sus narraciones. Por su parte, diez niños realizan transformaciones que tienen diferentes efectos, por lo que su incidencia es oscilatoria. Un menor número de niños mantienen sus textos para más de la mitad de las categorías y realizan cambios que tienen efectos negativos sobre el establecimiento de la coherencia. Los análisis permiten reconocer sujetos prototípicos de cada uno de los tipos de incidencia de los procesos de revisión que permiten caracterizar las formas de operar. En la Figura 1 se distinguen estos sujetos en color gris.

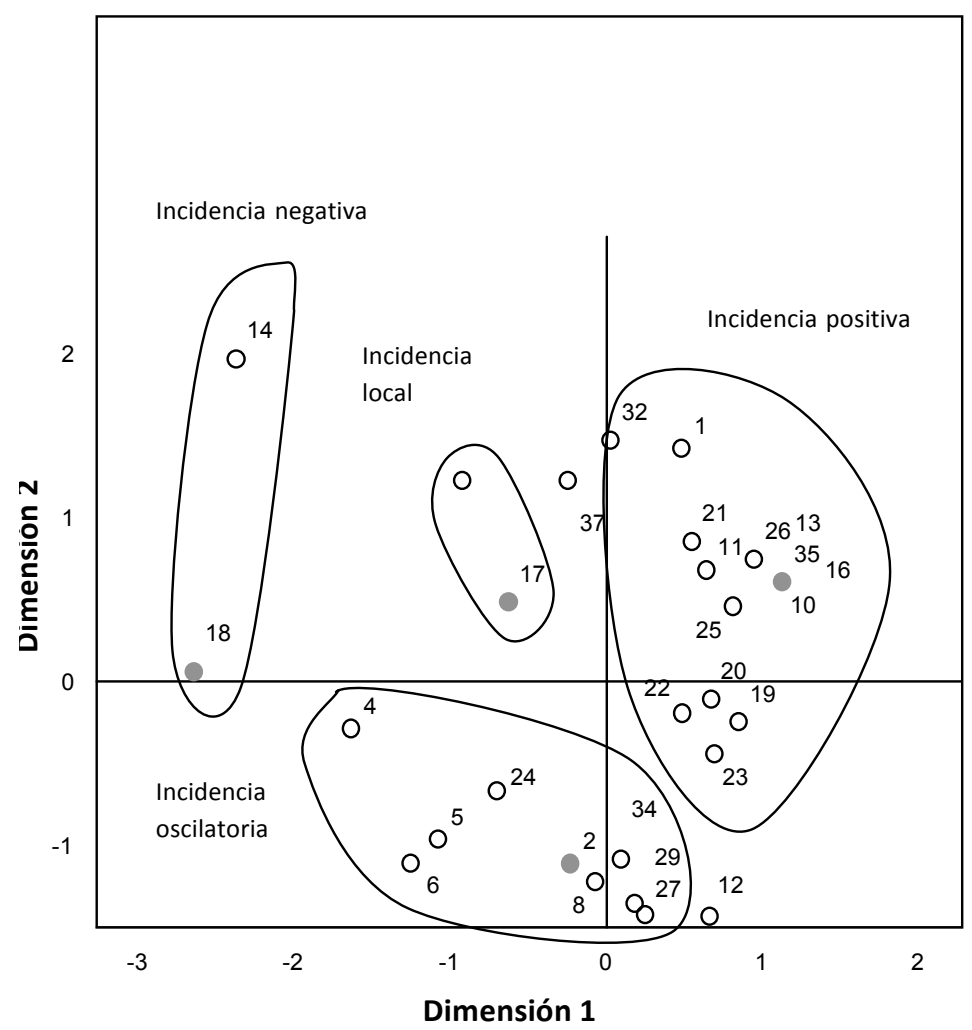

Figura 1. Análisis de correlación canónica no lineal para establecer grupos de niños según el nivel de incidencia de los procesos de revisión sobre la coherencia.

Fuente: elaboración propia. 
El estudio de los tipos de incidencia de los procesos de revisión sobre la coherencia en las narraciones, permite identificar diferencias sobre los marcos establecidos por los niños en sus textos, desde las primeras lecturas. Los escolares que han generado cambios con incidencia negativa y local tienden a focalizar su atención sobre aspectos puntuales: las palabras o su ortografía. En contraste en la incidencia oscilatoria y positiva se amplían estos marcos, y se considera la totalidad de la narración en relación con aspectos más particulares, en los que se centra la corrección -una palabra, un enunciado o una situación completa-.

El funcionamiento descrito está ligado a los recursos procedimentales escogidos por los niños para señalar aquello que quieren cambiar desde las primeras lecturas de sus propias producciones. Es decir, hay una relación entre los funcionamientos mentales puestos en marcha por los niños y las estrategias para establecer los marcos: subrayar una palabra, hacer señas sobre el enunciado por revisar o hacer guiones sobre las partes del texto que requieren mayor atención.

Las formas de operar de los niños desde las lecturas de su texto son fundamentales, dado que caracterizan los procesos de revisión y constituyen una guía para la textualización. En esta relación entre revisión y textualización se identifican diferencias de acuerdo con el tipo de incidencia que generan los cambios sobre el establecimiento de la coherencia. De este modo, los niños que realizan procesos con incidencia negativa y local, tienden a incluir las correcciones realizadas a su texto tal como fueron pensadas en el momento de la lectura, sin realizar nuevas transformaciones sobre el discurso o el contenido. A diferencia de aquellos que tienen una incidencia oscilatoria y positiva, que en el momento de la reescritura vuelven sobre su texto para establecer nuevos marcos de revisión, considerar otras opciones de corrección y tomar decisiones.

Los desempeños descritos muestran en los niños con incidencia negativa y local una tendencia hacia procesos más lineales en los que primero leen, después establecen los marcos de selección, generan la alternativa de corrección y transforman el escrito. En contraste, los niños con incidencia oscilatoria y positiva tienden hacia procesos más recursivos, ellos mantienen diferentes funcionamientos de forma simultánea, leen, establecen marcos, proponen opciones de corrección, se devuelven en el texto, consideran las opciones propuestas, avanzan, vuelven atrás. Además, el momento de la escritura se convierte en la continuación de identificación de disonancias, establecimiento de marcos, reflexión sobre estos aspectos, generación de opciones de corrección y transformación del escrito. Es decir, la toma de decisiones se mantiene a lo largo del proceso. Lo anterior da cuenta del lugar de la recursividad en los procesos de revisión, que tiene un efecto positivo en el establecimiento de la coherencia.

En este sentido, es importante recordar que recursividad viene del latín 'recurrere' que significa volver a correr (Perinat, 1995), por lo que implica la idea de un recorrido y la iteración de un proceso. Este funcionamiento lo manifiesta con claridad el sujeto prototípico de la incidencia positiva, en la forma en que procede sobre su texto. La Figura 2 muestra el recorrido del niño en el proceso de revisión de su texto, ilustrando las demarcaciones realizadas, las alternativas de corrección, la vuelta sobre la historia y el avance en el proceso.

Para representar el recorrido realizado por el niño se proponen flechas y números que facilitan seguir el orden en el que opera sobre el texto. Las flechas dan cuenta de los marcos de atención establecidos y de las alternativas de cambios consideradas, que permiten bosquejar el funcionamiento mental del niño. En particular, ilustran la recursividad del pensamiento que se refleja al volver sobre el texto ya leído, para incluir nuevas ideas y en las correcciones realizadas a las correcciones consideradas (en la Figura 2 se observan enunciados tachados que son reescritos y líneas realizadas por el niño que indican el lugar en el texto al que corresponden los cambios considerados). El material presentado se obtuvo durante el segundo conversatorio, al escolar se le facilitó un lapicero verde para señalar aquello que quería cambiar para mejorar su texto (el sujeto realiza guiones al inicio de la línea), luego uno azul para escribir sus nuevas ideas y después un lápiz negro para realizar la reescritura; los diferentes colores en la producción del niño ayudan 
a identificar el momento en el que se producen los cambios. Este seguimiento del proceso de revisión del niño, permite comprender el tipo de incidencia sobre la coherencia y los funcionamientos mentales implicados, interés central del estudio.

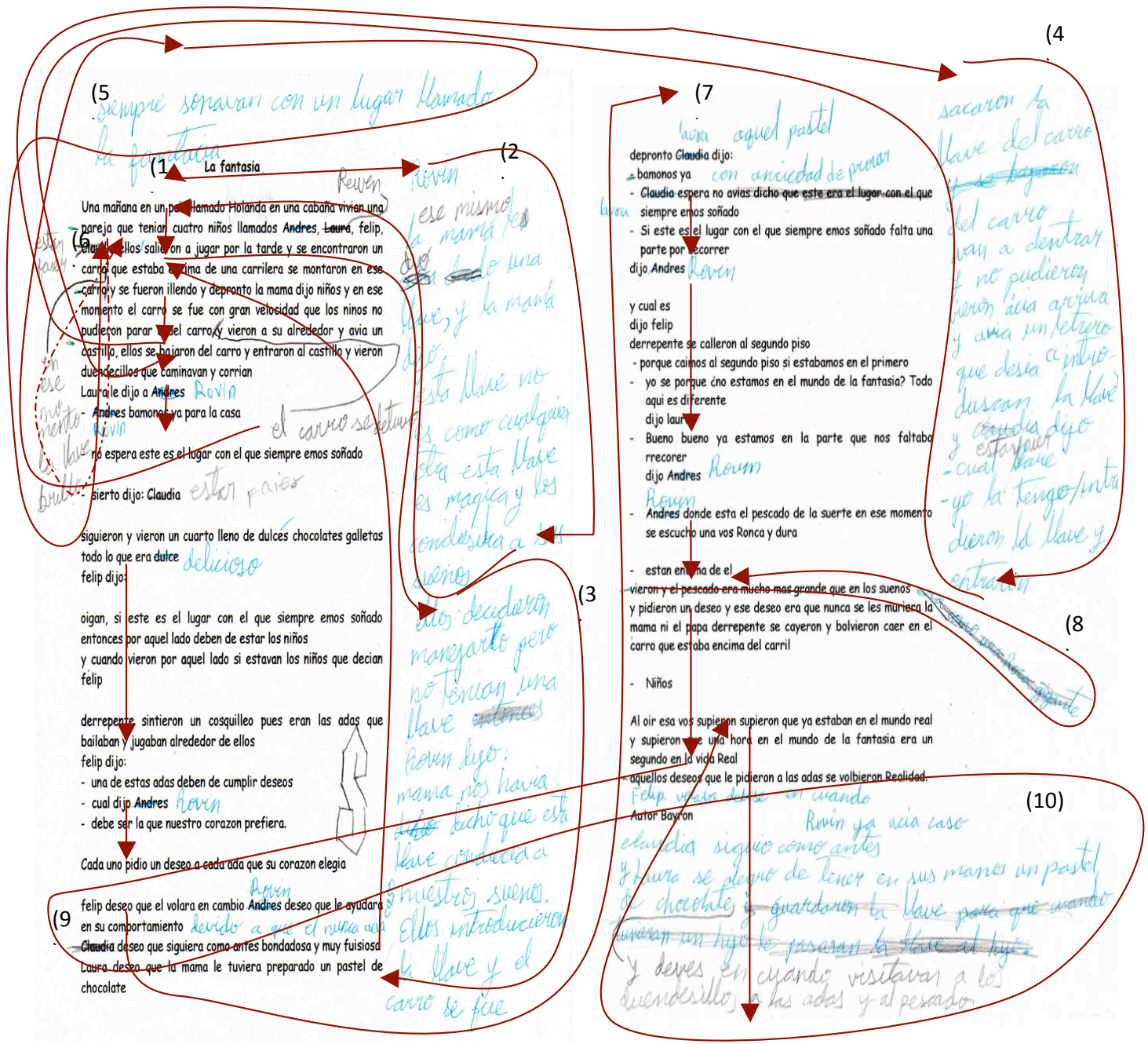

Figura 2. Recorrido realizado en la revisión de la segunda producción por el sujeto prototípico de la incidencia positiva. Fuente: elaboración propia.

Por su parte, el análisis indica que un énfasis en los cambios locales puede generar incongruencias. Cuando los niños logran mantener simultáneamente la mirada sobre lo local y aspectos más globales, al realizar cambios particulares identifican que deben modificar contenidos ligados con la situación para mantener la congruencia. Privilegiar lo global ofrece mayores posibilidades para mantener la con- gruencia del texto, en este caso los niños tienden a realizar ajustes en las reelaboraciones del escrito aun cuando algunas de sus estrategias de corrección resulten inadecuadas. En síntesis, en los procesos de revisión se establecen diferencias entre los sujetos que se centran en lo local, aquellos que logran combinar aspectos locales y globales de la historia, y quienes privilegian lo global desde el principio. 


\section{Discusión}

El análisis de las producciones textuales de los niños ha permitido identificar que los procesos de revisión ponen en marcha diferentes funcionamientos mentales implicados en el establecimiento de la coherencia a lo largo de las reescrituras. En esta investigación se resaltan los funcionamientos que siguen.

Primero, el doble papel de lector y escritor que implica funcionamientos distintos al de representarse a los lectores de un texto (Eco, 1996; Ochoa, Aragón, Correa \& Mosquera, 2008), a comprender un texto escrito por otro que requiere para la construcción de significado "la conciencia del lector y el monitoreo durante el proceso de comprensión" (Flórez et al., 2005, p. 20) y a situarse a sí mismo como lector del propio texto. Este funcionamiento alude a la posibilidad del autor de situarse en el rol de escritor en tanto productor de su texto y, cuando lee aquello que ha quedado consignado, distanciarse por momentos de su escrito. Algunos autores han resaltado la lectura como una actividad puesta en marcha durante la escritura, y diferenciado estrategias de acuerdo con el momento en el que se produce. Entre las estrategias identificadas está la lectura seguida de la generación de ideas y la lectura seguida de una revisión del texto (Rijlaarsdam, Couzijn \& Van Den Bergh, 2004). A diferencia del interés de estos autores sobre el momento en el que se realiza la lectura, en este documento se resaltan las exigencias que conlleva el hecho de situarse mentalmente en el rol de lector del propio texto.

El proceso de revisión posterior a un primer escrito implica que el sujeto se sitúe como lector de su narración. Este rol exige un distanciamiento cognitivo que lleva al autor a diferenciar entre lo que ha quedado consignado, lo que lee en voz alta y lo que quiso expresar. Algunos niños leen sus propias ideas y aquello que quisieron dejar plasmado en la narración, lo que se evidencia en diferentes situaciones: (a) la mayoría de escolares leen correctamente las palabras con la acentuación convencional, aunque en su texto falten letras, tildes o puntuación; (b) una niña, por ejemplo, lee la cantidad que ha pensado para su texto-quince millones- no aquella que ha quedado representada $-1.500 .000-$ y (c) algunos niños suprimen la reiteración y organizan la secuencia dentro del enunciado, cuando lo leen. Los niños no encuentran razones para establecer marcos durante la revisión, ni, en algunos casos, corregir los textos escritos, dado que no establecen distancia entre aquello que quieren decir y aquello que se dice.

Por su parte, otros niños reconocen la diferencia entre el texto y lo que quieren expresar, incluso algunos se sorprenden al hacerlo ("esta no la había visto antes"). Este distanciamiento entre la organización del contenido y el discurso que se quiere representar y la representación resultante, es uno de los primeros momentos del proceso de revisión que le permite a los sujetos hacer cambios en sus textos. Lo anterior no corresponde a lo propuesto por Bereiter y Scardamalia (1987), quienes plantean que los procesos de revisión empiezan cuando, al comparar el texto intentado y el texto actual, se detecta una desigualdad. Teniendo en cuenta que no se trata solo de una comparación, los conversatorios con los niños muestran que este doble papel mental de producir el texto, distanciarse y leerse, posibilita una toma de conciencia sobre aspectos que no habían sido considerados antes. Es decir, la revisión favorece la construcción textual, la posibilidad de organizar las ideas y descubrir nuevos elementos a tener en cuenta.

En cuanto a la representación como lector del propio texto, en los conversatorios de los niños, se reconocen diferentes criterios que guían los cambios. Algunos de ellos giran en torno a la información necesaria para que otros sujetos entiendan e interpreten correctamente la narración. De ahí, que los escolares argumenten: "Para que los niños lo entiendan es necesario aclarar que...", "Yo cambio esta expresión porque así es más comprensible para los niños", "Si no escribo esto no lo van a entender". La posibilidad de leer su propio texto y al mismo tiempo considerar a otros como lectores, ha permitido a los niños avanzar en la organización de la narración, en las relaciones establecidas entre los diferentes eventos y en la congruencia en las descripciones de los personajes. 
El segundo funcionamiento que se plantea son las evaluaciones de los niños en los procesos de revisión, Bereiter y Scardamalia $(1987,1992)$ y McCutchen (1995) han señalado que estas hacen parte de los procesos de revisión. En una de sus investigaciones Bereiter y Scardamalia (1987) proponen a los niños once frases evaluativas para que ellos escojan aquella que caracteriza su texto, y así facilitar operaciones de comparación entre el texto intentado y el actual, que conduzcan a detectar problemas. McCutchen (1995) afirma que la revisión involucra la lectura del texto, su evaluación y los cambios que sean necesarios. El estudio de la evaluación está ligado con el reconocimiento de inconsistencias en la composición. A diferencia de estas investigaciones, se reconoce y resalta el lugar de las evaluaciones en los procesos de revisión no solo como detección de problemas, sino considerándola desde la posibilidad de los niños de reflexionar sobre sus propios textos, independientemente de si estas reflexiones coinciden o no con lo que se espera que señalen como problemático.

El tercer funcionamiento es el establecimiento de marcos, los señalamientos y evaluaciones sobre el texto develan un funcionamiento mental en el que los niños pueden demarcar una parte, manteniendo de fondo la totalidad del texto, lo que indica que la mente trabaja simultáneamente sobre diferentes niveles; unos se actualizan otros se dejan suspendidos para retomarse en el momento requerido. En este sentido, lo propuesto por Perinat (1995) resulta esclarecedor "trazar un marco es análogo a delimitar un subconjunto dentro de un conjunto" (p. 194). Para Perinat este establecimiento de marcos está ligado a la recursividad, dado que él entiende este funcionamiento como incrustración, establecimiento de paréntesis, acotación o frames. Este último término inglés es retomado por Perinat de Gregory Bateson (1955/1972) equivalente a 'marco', resultado de la acción de enmarcar (p. 193). Continua Perinat "un bucle recursivo traza un "marco" o demarcación; acota un subsistema dentro del sistema principal. Pero, a la vez, un bucle recursivo mantiene la conexión entre el subespacio enmarcado y el espacio en que se ha delimitado el marco" (p. 193). Este funcionamiento es identificado en los niños que logran realizar procesos de revisión que los llevan a generar cambios con incidencia en el establecimiento de la coherencia.

Las evaluaciones hechas sobre los textos apuntan tanto a las formas de expresión como a los contenidos apropiados. Estas evaluaciones actualizan el saber enciclopédico (Eco, 1996) de los niños ligado con su propuesta textual y con las nuevas reflexiones realizadas, al tiempo que llevan a construir criterios que rigen las modificaciones en la historia. Se podría pensar que dichas evaluaciones tienen como único objetivo mejorar la calidad textual, sin embargo, es interesante descubrir las diferentes razones que movilizan a los niños. Algunas evaluaciones se rigen por la correspondencia con el gusto personal; en otros casos, los niños buscan que la historia concuerde con el sistema de valores que han construido tanto ligado al deber ser como a sus propias reflexiones. De este modo, se puede distinguir entre el objeto de la evaluación -el discurso y el contenido- y los criterios que rigen las modificaciones consideradas.

La evaluación sobre el texto se muestra de forma reiterativa en los procesos de los niños como un aspecto fundamental en la revisión. Se puede diferenciar entre dos tipos de evaluación: (a) valoraciones sobre el texto, que favorecen el establecimiento de marcos dentro de la totalidad de la historia y llevan a identificar aspectos que deben ser reconsiderados y/o transformados y (b) juicios sobre las opciones de corrección, que involucran una toma de conciencia entre la distancia del contenido a representar y las formas en que puede ser representado (De Castro, 2008).

En síntesis, las evaluaciones realizadas pueden regirse por diferentes criterios establecidas por los niños durante los procesos de revisión. Así, los criterios que resaltan el conocimiento del autor sobre el mundo en función de la historia propuesta, posibilitan el avance en la verosimilitud. Los razonamientos que enfatizan sobre la organización textual y la información que requiere un lector externo para comprender la historia, favorecen el avance en la coherencia. Los criterios desde aspectos externos al texto que relegan la historia, pueden llevar a incongruencias. En conjunto, las razones 
subyacentes a las transformaciones en los textos pueden desligarse de la coherencia, aunque los cambios realizados puedan afectarla tanto positiva como negativamente. De ahí, la importancia de la toma de conciencia sobre los criterios que rigen los cambios propuestos en los textos.

Los desempeños de los niños descritos hasta el momento permiten destacar la distancia que existe entre identificar qué cambiar y saber cómo hacerlo. Los procesos de revisión no necesariamente se materializan en cambios en la composición escrita (Bereiter \& Scardamalia, 1987; Camps, 1992; Rouiller, 2004). En esta dirección, se distingue entre identificar qué cambiar y saber cómo hacerlo. Algunos niños identifican aspectos que son disonantes en relación con sus ideas sobre la historia, ese hecho genera la pregunta sobre cómo cambiar el texto. Modificarlo encierra una exigencia cognitiva, porque obliga a mantener el criterio y enfrentar los problemas que propone la escritura. En este momento, algunos niños sienten que es demasiado difícil y deciden dejar el texto tal como está, situación que ejemplifica una niña cuando tacha un segmento que desea suprimir en la reescritura, sin embargo, no encuentra cómo organizar nuevamente su composición y mantiene su escrito. En una sesión posterior, durante el segundo conversatorio, la niña vuelve a intentar eliminar el mismo fragmento, lo señala y logra efectuar el cambio.

Surge entonces la pregunta sobre cuáles son las exigencias cognitivas que impone saber cómo transformar el texto después del establecimiento del marco. La niña sabe que quiere suprimir un segmento y lo tacha, sin embargo, el problema que enfrenta es cómo volver a conectar su texto, porque suprimir una parte implica reorganizar la red de significados que establecen las relaciones en la historia. Lo descrito da cuenta de la estrecha relación entre saber qué modificar, cómo hacerlo y el establecimiento de la coherencia. De ahí, que algunos niños realicen transformaciones que afectan negativamente la coherencia, al mantener la atención sobre una parte y descuidar la totalidad, aun cuando sus criterios para modificar un segmento sean adecuados.

En los funcionamientos puestos en marcha en la revisión es importante diferenciar entre la re- presentación del texto, la posibilidad de establecer marcos para focalizar la atención, la búsqueda de estrategias para realizar cambios y reestablecer la red de relaciones y que los cambios realmente resuelvan el problema considerado. Un patrón que se presenta de forma regular en los niños durante los conversatorios y las reescrituras, es que vuelven varias veces sobre un mismo marco que ya ha sido establecido. Esto indicaría una conciencia en los escritores sobre la distancia entre saber qué cambiar, cómo hacerlo y cuál sería la mejor alternativa de cambio.

El desarrollo de esta investigación permite entender la coherencia como una red de significados que establece relaciones y articula una historia escrita por los niños. También permite comprender que los procesos de revisión tienen una incidencia en la coherencia, en particular cuando se producen cambios en las conexiones entre las diferentes situaciones de la narración. La coherencia está lejos de ser una cuestión dicotómica coherente-incoherente, se trata de un funcionamiento que realiza el autor en el que construye enlaces entre los diferentes eventos que configuran la historia.

Para finalizar, es pertinente detenerse a precisar los aportes de la investigación, en torno a la Psicología del desarrollo cognitivo interesada en los procesos de escritura. El diseño compuesto por reescrituras y conversatorios sobre los textos es un procedimiento eficaz, que permite el estudio de funcionamientos puestos en marcha en la composición textual, y no solo del producto final. El conjunto de la información que surge de esos procedimientos y la articulación de los análisis cualitativos y cuantitativos, permiten realizar un seguimiento sobre los procesos de revisión y la incidencia que tienen en el establecimiento de la coherencia.

En el trabajo, se identifican diferentes tipos de incidencia en los procesos de revisión sobre el establecimiento de la coherencia en las reescrituras de textos narrativos: positiva, oscilatoria, local y negativa. También se reconoce que la mayoría de los niños emprende procesos de revisión que les permiten avanzar en la organización del texto; estos avances en los trabajos de reescritura varían en grado. De igual manera, se identifican procesos 
caracterizados por cambios en el escrito, que tienen una incidencia oscilatoria sobre la coherencia y que representan una vía alterna para avanzar en la producción escrita.

Los resultados permiten identificar funcionamientos cruciales en el estudio de los procesos de escritura: situarse como lector de su texto, lograr un distanciamiento que lo lleve a diferenciar entre lo que ha quedado consignado, lo que le suscita la lectura y lo que quiere representar; funcionamientos que pueden ser profundizados en futuras investigaciones. De igual modo, la propuesta es seguir estudiando las evaluaciones del escritor sobre el propio texto ligado a los funcionamientos metacognitivos.

Otro tipo de aportes derivados de la investigación se relacionan con la educación y las prácticas propuestas a los alumnos, para la enseñanza de la lengua materna en la escuela. En este sentido, es importante fomentar el trabajo de reescrituras en el aula de clase. Las reescrituras, si bien no determinan un progreso en la producción textual, posibilitan al sujeto tomar conciencia sobre el texto, reconsiderar las decisiones y representarse nuevos funcionamientos textuales que sean coherentes con la historia desarrollada.

Además, las reescrituras permiten asumir diferentes momentos para enfrentar las exigencias de la producción textual. De ahí que la mayoría de los niños se centren en un aspecto a reelaborar durante una reescritura, situación que podría aprovecharse en el aula con propuestas que focalicen sobre un aspecto problemático identificado en la composición. Volver sobre el texto, reconsiderar las ideas y las formas en que se representa, favorecen una perspectiva analítica y crítica sobre las propias ideas y el discurso utilizado, así como sobre las posiciones de los otros y las formas como se materializan.

Por último, es relevante plantear estrategias educativas que reconozcan la revisión como un proceso inherente en la composición textual (Camps, 1992), distanciándola de la connotación negativa que la ubica desde el error y la sanción. Señalar el error para ser corregido es una práctica que ha demostrado resultar inadecuada, dado que los sujetos mantienen el mismo tipo de errores en sus escritos siguientes. Es cuando el sujeto establece criterios ligados a la organización del texto, identifica la existencia de diferentes alternativas para expresar un contenido y privilegia las conexiones en su composición, cuando se permean los procesos de escritura y logran un impacto a largo plazo. De ahí, la importancia de fomentar procesos de revisión sobre la coherencia en contextos educativos y no solo sobre aspectos notacionales u ortográficos, como generalmente se hace (De Castro, 2010). Estos resultados están en consonancia con la propuesta de Pérez-Abril (2003) "escribir va mucho más allá de transcribir, y las prácticas pedagógicas deben orientarse desde un concepto de escritura complejo" (p. 10).

Los resultados de la investigación constituyen un aporte a la comprensión global de la escritura en el país. La evaluación masiva en Colombia, liderada por el Sistema Nacional de Evaluación de la Educación SNE (SABER) y el Ministerio de Educación Nacional, ha permitido identificar entre las problemáticas en producción textual en educación básica primaria, la dificultad para componer textos completos, dada la tendencia a escribir oraciones o fragmentos, y el establecimiento de relaciones claras entre una idea y otra a través del establecimiento de enlaces propios del texto escrito (Pérez-Abril, 2003). Estos aspectos han sido analizados en este texto, desde funcionamientos cognitivos implicados en los procesos de composición, aportando nuevos elementos para la reflexión a nivel nacional.

\section{Referencias}

Allal, L. \& Chanquoy, L. (2004). Revision revisited [Introduction]. En G. Rijlaarsdam (Ed. de la serie) \& L. Allal, L. Chanquoy \& P. Largy (Eds. del volumen), Studies in writing: Vol. 13. Revision. Cognitive and instructional processes (pp. 1-8). Norwell, MA: Kluwer Academia Press.

Bereiter, C. \& Scardamalia, M. (1987). The psychology of written composition. Hillsdale, NJ: Erlbaum.

Bereiter, C. \& Scardamalia, M. (1992). Dos modelos explicativos de los procesos de composición escrita. Infancia y Aprendizaje, 58, 43-64.

Bigas, M., Clariana, M., Guasch, O., Luna, X., Milian, M. \& Ribas, T. (1994). La lengua escrita en la es- 
cuela: el texto narrativo a los ocho años. Infancia y Aprendizaje, 65, 75-101.

Blanche-Benveniste, C. (1998). Estudios lingüísticos sobre la relación entre oralidad y escritura. Barcelona: Gedisa.

Bourdin, B. \& Fayol, M. (2002). Even in adults, written production is still more costly than oral production. International Journal of Psychology, 37(4), 219-227.

Bortolussi, M. \& Dixon, P. (2003). Psychonarratoly. Foundations for the empirical study of literary response. Cambridge: Cambridge University Press.

Bruner, J. (1988). Realidad mental y mundos posibles. Los actos de la imaginación que dan sentido a la experiencia. Barcelona: Gedisa.

Cain, K. (2003). Text comprehension and its relations to coherence and cohesion in children's fictional narratives. British Journal of Developmental Psycho$\log y, 21,335-351$.

Calsamiglia, H. \& Tusón, A. (1999). Las cosas del decir. Manual de análisis del discurso. Barcelona: Ariel.

Camps, A. (1992). Algunas observaciones sobre la capacidad de revisión de los adolescentes. Infancia y Aprendizaje, 58, 65-81.

Cassany, D. (1996). La cocina de la escritura. Barcelona: Anagrama.

Cassany, D. (1997). Describir el escribir: cómo se aprende a escribir. Barcelona: Paidós.

De Castro, D. (2008). Los procesos cognitivos de revisión en el establecimiento de la coherencia en narraciones escritas por niños de 10 años. Tesis de maestría, Universidad del Valle, Cali, Colombia.

De Castro, D. (2010). Los procesos cognitivos de revisión en la producción textual. Revista Textos \& Sentidos, 2, 9-30.

De Vega, M. \& Cuetos, F. (1999). Psicolingüística del español. Madrid: Trotta.

Eco, H. (1996). Seis paseos por los bosques narrativos. Barcelona: Lumen.

Flórez, R., Torrado, M., Arévalo, I., Mesa, C., Mondragón, S. \& Pérez, C. (2005). Habilidades metalingüísticas, operaciones metacognitivas y su relación con los niveles de competencia en lectura y escritura: un estudio exploratorio. Forma y Función, 18, 15-44.
Flower, L. \& Hayes, J. (1996). Teoría de la redacción como proceso cognitivo. En R. Rudell, M. Rapp Rudell \& H. Singer (Eds.), Textos en contexto. Los procesos de lectura y escritura (Vol. 1, pp. 73-107). Buenos Aires: Asociación Internacional de Lectura.

Graham, S., Harris, K. \& Mason, L. (2005). Improving the writing performance, knowledge, and selfefficacy of struggling young writers: The effects of self-regulated strategy development. Contemporary Educational Psychology, 30, 207-241.

Hickmann, M. \& Schneider, P. (2000). Cohesion and coherence anomalies and their effects on children's referent introduction in narrative retell. En M. Perkins \& S. Howard (Eds.), New directions in language development and disorders (pp. 251-260). New York: Kluwer Academic/Plenum Publishers.

Levy, E. (2003). The roots of coherence in discourse. Human Development, 46, 169-188.

Martínez, M. (1994). Análisis del discurso. Cohesión, coherencia y estructura semántica de los textos expositivos. Cali, Col: Editorial Facultad de Humanidades, Universidad del Valle.

McCutchen, D. (1995). Cognitive processes in children's writing: Developmental and individual differences. Issues in Education, 1(2), 123-160.

Miras, M. (2000). La escritura reflexiva. Aprender a escribir y aprender acerca de lo que se escribe. Infancia y Aprendizaje, 89, 65-80.

Ochoa, S., Aragón, L., Correa, M. \& Mosquera, S. (2008). Funcionamiento metacognitivo de niños escolares en la escritura de textos narrativos, antes y después de una pauta de corrección conjunta. Acta Colombiana de Psicología, 11(2), 77-89.

Olson, D. (1998). El mundo sobre el papel. El impacto de la escritura y la lectura en la estructura del conocimiento. Barcelona: Editorial Gedisa.

Ong, W. (1999). Oralidad y escritura. Tecnologías de la palabra. Bogotá: Fondo de Cultura Económica.

Orozco, B. \& Correa, M. (2001). Investigación, intención y conflicto en la escritura de textos narrativos: un estudio con niños de 8-10 años (Informe técnico). Cali, Col: COLCIENCIAS.

Orozco, B., Correa, M., Conde, E. \& Hurtado, R. (2002). Relación contractual narrador-narratario en la escritura de textos narrativos: un estudio con niños de 8-10 
años (Informe técnico de la investigación). Cali, Col: COLCIENCIAS.

Pérez-Abril, M. (2003). Leer y escribir en la escuela. Algunos escenarios pedagógicos y didácticos para la reflexión. Bogotá: Instituto Colombiano para la Evaluación de la Educación (ICFES), Ministerio de Educación Nacional.

Perinat, A. (1995). Prolegómenos para una teoría del juego y del símbolo. Cognitiva, 7(2), 185-204.

Rijlaarsdam, G., Couzijn, M. \& Van Den Bergh, H. (2004). The study of revision as a writing process and as a learning-to-write process. En G. Rijlaarsdam (Ed. de la serie) \& L. Allal, L. Chanquoy \& P. Largy (Eds. del volumen), Studies in writing: Vol. 13. Revision. Cognitive and instructional processes (pp. 189-208). Norwell, MA: Kluwer Academia Press.
Rouiller, Y. (2004). Collaborative revision and metacognitive reflection in a situation of narrative text production. En G. Rijlaarsdam (Ed. de la serie) \& L. Allal, L. Chanquoy \& P. Largy (Eds. del volumen), Studies in writing: Vol. 13. Revision. Cognitive and instructional processes (pp. 171-188). Norwell, MA: Kluwer Academia Press.

Severinson Eklundh, K. \& Kollberg, P. (1996). A computer tool and framework for analyzing on-line revisions. En C. Levy \& S. Ransdell (Eds.), The science of writing: Theories, methods, individual differences, and applications (pp. 163-188). Mahwah, NJ: Erbaum.

Teberosky, A. (1989). La escritura de textos narrativos. Infancia y Aprendizaje, 46, 17-35.

Van Allsburg, C. (1999). Los misterios del señor Burdick. México: Fondo de Cultura Económica. 


\section{Apéndice}

Fuente generativa para recolección de información

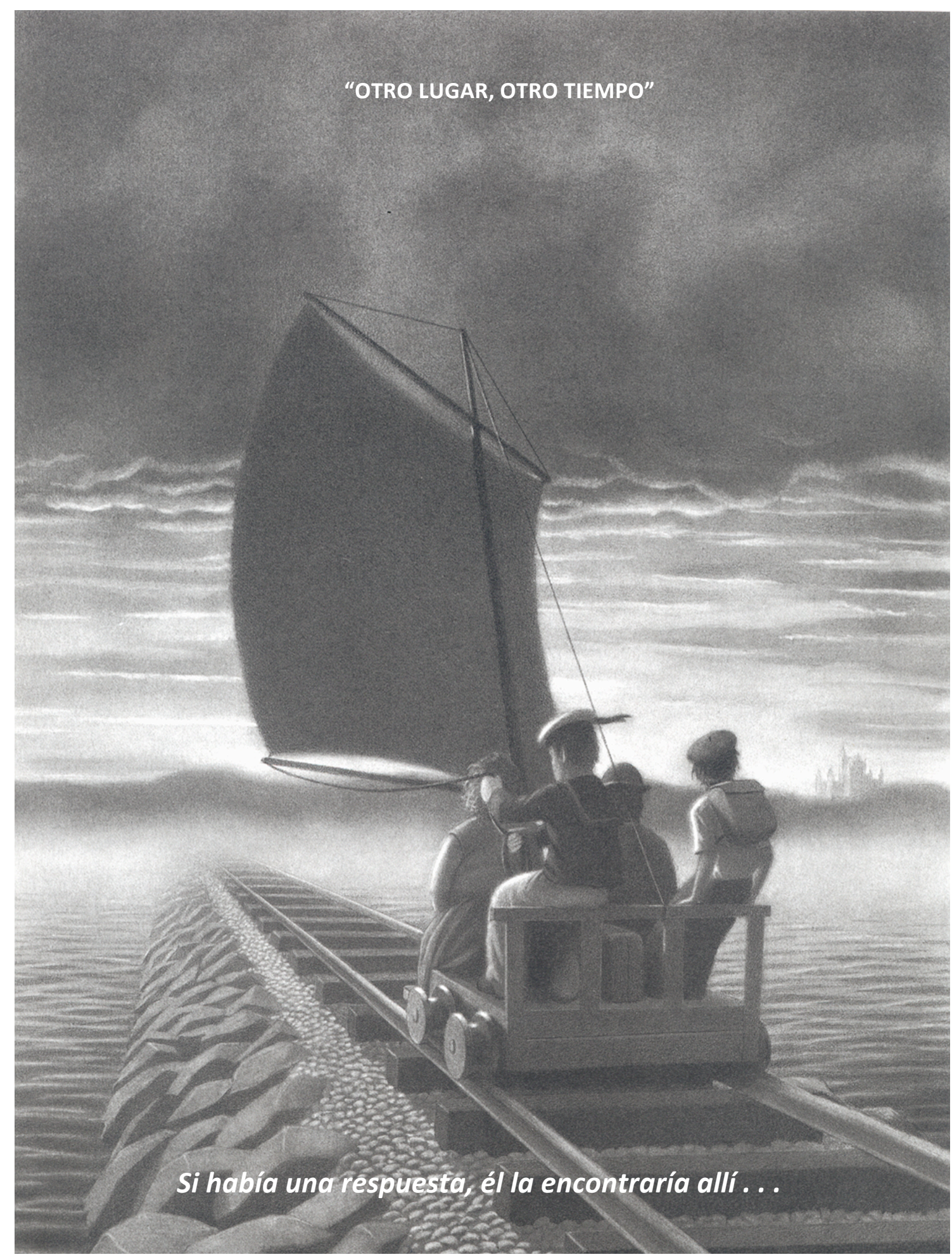

